\title{
ON BARRELLING FOR A SPECIAL MATERIAL IN FINITE ELASTICITY*
}

BY

HENRY C. SIMPSON (University of Tennessee)

AND

SCOTT J. SPECTOR (University of Minnesota and Southern Illinois University)

Introduction. We herein continue our investigation of the stability of a solid circular cylinder, composed of a particular homogeneous isotropic (compressible) nonlinearly elastic material, that is subjected to compressive end forces in the direction of its axis (so as to give fixed axial displacements at the ends) ${ }^{1}$.

The particular material used in this analysis has stored energy function $\sigma$ given by ${ }^{2}$

$$
\sigma(\mathbf{F})=\frac{1}{2} \mathbf{F} \cdot \mathbf{F}+\frac{1}{m}(\operatorname{det} \mathbf{F})^{-m}
$$

for some $m>0$ and all $3 \times 3$ matrices F (Blatz \& Ko [3] have shown that, with $m=13.3$, this material can be matched to experimental data of Bridgman [4]).

We begin our analysis with a formulation of the relevant non-linear boundary-value problem and a construction of a trivial homogeneous solution to this problem. We then consider the superimposed linear (infinitesimal) problem ${ }^{3}$ about a given solution of the nonlinear problem.

We first note that a result of Simpson and Spector [9] shows that all solutions ${ }^{4}$ of the linear problem, sans the traction free boundary condition on the curved surface of the cylinder, can be obtained by separation of variables. Thus the question of stability reduces to proving that a certain determinant, that corresponds to the traction free condition, is zero at some critical value of the loading.

\footnotetext{
* Received by the editors April 3, 1983.

'Experiments of Beatty \& Hook [2] and Beatty \& Dadras [1] have shown that such cylinders retain their cylindrical shape until a certain critical value of the loading is reached. Further loading of the cylinder results in buckl ing for thin cylinders and axisymmetric bulging (barrelling) for thick cylinders.

${ }^{2}$ Burgess \& Levinson [5] have used this material in their analysis of biaxial compression of rectangular rods.

${ }^{3}$ The first such analysis was done by Wilkes [11], who assumed that the cylinder was infinite and composed of an incompressible material.

${ }^{4}$ This is a crucial point that (to our knowledge) has not been addressed in the literature. If an additional solution, not obtainable by separation of variables, exists this solution might satisfy the remaining boundary condition at a smaller value of the loading than the separation of variables solution.
} 
We then show that for each eigenmode $n=1,2,3, \ldots$, there exists a unique loading value $\lambda(n)$ that makes the above mentioned determinant zero ${ }^{5}$ and hence conclude that the cylinder will eventually become unstable when compressed in an axial direction.

Our main results concern the dependence of the loading value $\lambda$ (where instability occurs) on the parameter $n$. These results allow us to conclude that:

(i) for a given value of $\lambda$ at most two eigenmodes can occur;

(ii) for sufficiently thick cylinders $n=1$ is the eigenmode that occurs first ${ }^{6}$;

(iii) for sufficiently thin cylinders $n=1$ is not the eigenmode that occurs first;

(iv) there exist cylinders whose first point of instability occurs at two distinct eigenmodes simultaneously.

Finally, we use numerical techniques to obtain a global picture of the dependence of $\lambda$ upon $n$.

1. Notation. We let

$$
\text { Lin } \equiv \text { space of all linear transformations from } \mathbf{R}^{3} \text { into } \mathbf{R}^{3}
$$

with inner product

$$
\mathbf{G} \cdot \mathbf{H}=\operatorname{trace}\left(\mathbf{G H}^{T}\right),
$$

where $\mathbf{H}^{T}$ is the transpose of $\mathbf{H}$ and denote

$$
\operatorname{Lin}^{+}=\{\mathbf{H} \in \operatorname{Lin}: \operatorname{det} \mathbf{H}>0\},
$$

where det is the determinant.

We denote by $I_{n}(t)$ the modified Bessel functions of the first kind, so that $I_{n}$ are globally analytic solutions of the differential equation

$$
t^{2} \ddot{I}_{n}(t)+t \dot{I}_{n}(t)-\left(t^{2}+n^{2}\right) I_{n}(t)=0,
$$

and are given by

$$
I_{n}(t)=\sum_{r=0}^{\infty} \frac{(t / 2)^{n+2 r}}{r !(n+r) !}
$$

This infinite series formula can be used to prove that $I_{0}$ and $I_{1}$ satisfy the recurrence relations

$$
\dot{I}_{0}(t)=I_{1}(t), \quad t \dot{I}_{1}(t)=t I_{0}(t)-I_{1}(t)
$$

It can be shown that $I_{0}$ and $I_{1}$ have asymptotic expansions

\footnotetext{
${ }^{5}$ In [9] we prove the existence of such a loading value for a larger class of materials, the Hadamard-Green materials. The existence analysis in this paper is a special case of that result.

${ }^{6}$ This contrasts with results of Sawyers \& Rivlin [7] and Sawyers [8] for incompressible materials. Their analysis indicates that $\lambda$ increases with $n$ for barrelling and hence the first mode is $n=\infty$. This is thought to be exhibited physically as surface wrinkling.

${ }^{7} \mathrm{Cf}$. Copson [6], pp. 72-75 where asymptotic expansions for $J_{0}$ and $J_{1}$ are rigorously derived using Watson's Lemma.
} 


$$
\begin{aligned}
& \sqrt{2 \pi t} e^{-t} I_{0}(t)=1+\frac{1}{8 t}+\frac{9}{128 t^{2}}+O\left(\frac{1}{t^{3}}\right) \\
& \sqrt{2 \pi t} e^{-t} I_{1}(t)=1-\frac{3}{8 t}-\frac{15}{128 t^{2}}+O\left(\frac{1}{t^{3}}\right)
\end{aligned}
$$

as $t \rightarrow \infty$.

Some of the results in this paper specifically deal with the function

$$
v(t) \equiv t I_{0}(t) / I_{1}(t) \text {. }
$$

A simple consequence of the recurrence relations (1.2) and the infinite series (1.1) is that $v$ satisfies

$$
t \dot{v}(t)=t^{2}+2 v(t)-v^{2}(t), \quad v(0)=2 .
$$

One can also use (1.3) and (1.5) to establish the asymptotic estimates

$$
\begin{array}{ll}
v(t)=t+\frac{1}{2}+a(t), & \operatorname{Lim}_{t \rightarrow \infty} t a(t)=\frac{3}{8}, \\
\dot{v}(t)=1+b(t), & \operatorname{Lim}_{t \rightarrow \infty} t b(t)=0 .
\end{array}
$$

Finally, we will make use of the following results.

Proposition $1.1[10]$.

(i) $\dot{v}(t)$ is strictly positive for $t \in(0, \infty)$;

(ii) $\ddot{v}(t)$ is strictly positive for $t \in[0, \infty)$;

(iii) $v^{2}(t)-\left(t^{2}+2\right)>0$.

2. The constitutive relation. The non-linear problem. We consider a homogeneous body $\mathscr{B}$ and identify $\mathscr{B}$ with the region (of $\mathbf{R}^{3}$ ) it occupies in a fixed homogeneous reference configuration. A deformation $f$ of the body is a member of the space

$$
\text { Def }=\left\{\mathbf{f} \in C^{2}\left(\mathscr{B}, \mathbf{R}^{3}\right): \operatorname{det} \nabla \mathbf{f}>0\right\} .
$$

We assume that the body in the reference configuration is in the shape of a right circular cylinder of height $L$ and radius $R$. In rectangular coordinates we take

$$
\mathscr{B}=\left\{\left(x_{1}, x_{2}, x_{3}\right): x_{1}^{2}+x_{2}^{2} \leqslant R^{2}, x_{3} \in[0, L]\right\}
$$

with lateral surface

$$
\mathcal{S}=\left\{\left(x_{1}, x_{2}, x_{3}\right): x_{1}^{2}+x_{2}^{2}=R^{2}, x_{3} \in[0, L]\right\} .
$$

The remaining two pieces of $\partial \mathscr{B}$, the top and bottom of the cylinder, we denote by $\mathcal{C}_{T}$ and $e_{B}$, respectively.

We assume that $\mathscr{B}$ is hyperelastic with (Piola-Kirchhoff) stress $\mathbf{S}: \operatorname{Lin}^{+} \rightarrow$ Lin given by

$$
\mathbf{S}(\mathbf{F})=\mathbf{F}-(\operatorname{det} \mathbf{F})^{-m} \mathbf{F}^{-T}
$$

\footnotetext{
${ }^{8}$ We note that we only use this constitutive relation for linear transformations whose determinant is less than or equal to one.
} 
while the linear transformation $\mathbf{A}(\mathbf{F})$ : Lin $\rightarrow$ Lin defined by

$$
\mathbf{A}(\mathbf{F}) \mathbf{H} \equiv\left(\frac{d}{d \mathbf{F}} \mathbf{S}(\mathbf{F})\right) \mathbf{H}=\mathbf{H}+(\operatorname{det} \mathbf{F})^{-m}\left[m\left(\mathbf{F}^{-T} \cdot \mathbf{H}\right) \mathbf{F}^{-T}+\mathbf{F}^{-T} \mathbf{H}^{T} \mathbf{F}^{-T}\right]
$$

is called the elasticity tensor.

Let $\lambda \in(0,1]$ and consider the problem of finding a deformation $\mathbf{f}$ that satisfies

$$
\begin{array}{ll}
\operatorname{div} \mathbf{S}(\nabla \mathbf{f})=\mathbf{0} & \text { in } \Re_{,}, \\
f_{3}=0 & \text { on } \mathcal{C}_{B}, \\
f_{3}=\lambda L & \text { on } \mathcal{C}_{T}, \\
S_{13}(\nabla \mathbf{f})=S_{23}(\nabla \mathbf{f})=0 & \text { on } \mathcal{C}_{B} \text { and } \mathcal{C}_{T}, \\
\mathbf{S}(\nabla \mathbf{f}) \mathbf{n}=\mathbf{0} & \text { on } \mathcal{S},
\end{array}
$$

where $\mathbf{n}$ is the outward unit normal to the lateral surface and the parameter $\lambda$ is the ratio of the final to the initial height of the cylinder.

In view of our choice of constitutive relation it is easy to show that (2.3) has a unique (up to a rigid deformation) trivial homogeneous solution

$$
\mathbf{f}_{\lambda}(\mathbf{x})=\left(\begin{array}{lll}
\mu^{1 / 2} & & \\
& \mu^{1 / 2} & \\
& & \lambda
\end{array}\right) \mathbf{x}, \quad \mu=\lambda^{-m /(m+1)} .
$$

3. The linear problem. We now investigate the stability of the non-linear solution by linearizing the boundary-value problem. For a given $\lambda \in(0,1]$ and $\mathbf{f}_{\lambda}$ we linearize (2.3) to arrive at the problem of finding a nontrivial (cf. [9]) $C^{2}$ function $\mathbf{u}$ that satisfies

$$
\begin{array}{ll}
\operatorname{div} \mathbf{A}[\nabla \mathbf{u}]=\mathbf{0} & \text { in } \mathscr{B}, \\
u_{3}=0 & \text { on } \bigodot_{B} \text { and } \mathcal{C}_{T}, \\
\mathbf{A}[\nabla \mathbf{u}]_{13}=\mathbf{A}[\nabla \mathbf{u}]_{23}=0 & \text { on } \bigodot_{B} \text { and } \bigodot_{T}, \\
\mathbf{A}[\nabla \mathbf{u}] \mathbf{n}=\mathbf{0} & \text { on } \mathcal{S},
\end{array}
$$

where $\mathbf{A}=\mathbf{A}\left(\nabla \mathbf{f}_{\lambda}\right)$ and once again $\mathbf{n}$ is the outward unit normal to the lateral surface.

For the remainder of this paper we will only be interested in considering specific types of instabilities, those that are axisymmetric. We shall therefore consider linear solutions of the form

$$
\mathbf{u}\left(x_{1}, x_{2}, x_{3}\right)=\left(\begin{array}{c}
\phi(r, z) x_{1} \\
\phi(r, z) x_{2} \\
l(r, z)
\end{array}\right)
$$

where $r^{2}=x_{1}^{2}+x_{2}^{2}$ and $z=x_{3}$.

If we let $\theta(r, z) \equiv r^{2} \phi(r, z)$ a straightforward computation, using (2.2) and (2.4), shows that (3.1) reduces to

$$
(m+2)\left(\theta_{r} / r\right)_{r}+\theta_{z z} / r+t^{1 / 2}(m+1) l_{r z}=0 \quad \text { on }(0, R] \times[0, L],
$$




$$
\begin{array}{rc}
\left(r l_{r}\right)_{r}+[1+t(m+1)] r l_{z z}+t^{1 / 2}(m+1) \theta_{r z}=0 & \\
l(r, 0)=l(r, L)=\theta_{z}(r, 0)=\theta_{z}(r, L)=0 & \text { for } r \in(0, R], \\
R l_{r}(R, z)+t^{1 / 2} \theta_{z}(R, z)=0 & \text { for } z \in[0, L] . \\
(m+2) \theta_{r}(R, z)+t^{1 / 2} m R l_{z}(R, z)=2 \theta(R, z) / R &
\end{array}
$$

where $t \equiv \mu \lambda^{-2}$. In addition the requirement $\mathbf{u} \in C^{2}$ yields

$$
\theta(r, z) / r \rightarrow 0 \text { as } r \rightarrow 0
$$

uniformly in $z$.

Proposition 3.1 [9]. Let $\lambda \in(0,1)$. Then any $C^{2}$ solution $(\theta, l)$ of $(3.2),(3.3)$, and (3.5) can be written

$$
\theta(r, z)=\sum_{n=1}^{\infty} \theta_{n}(r) \cos \left(\rho_{n} z\right), \quad l(r, z)=\sum_{n=1}^{\infty} l_{n}(r) \sin \left(\rho_{n} z\right) .
$$

where $\rho_{n}=n \pi / L ; \theta_{n}, l_{n}$ are linear combinations of

$$
\theta_{n i}(r)=r I_{1}\left(\rho_{n} r e_{i}\right), \quad l_{n i}(r)=D_{i} I_{0}\left(\rho_{n} r e_{i}\right),
$$

$i=1,2 ;$ and

$$
\begin{gathered}
e_{1}=1, \quad e_{2}^{2}=1+(t-1) \frac{m+1}{m+2}, \\
D_{1}=-t^{-1 / 2}, \quad D_{2}=-t^{1 / 2} / e_{2} .
\end{gathered}
$$

We next want to show that there is a $\lambda \in(0,1)$ such that our linear problem, in the form (3.2)-(3.5), has a non-trivial solution. Clearly, Proposition 3.1 reduces this problem to that of finding a $\lambda$ such that one of the solutions $(\theta, l)$ to (3.2), (3.3), and (3.5) satisfies the boundary condition on the lateral surface (3.4). We note that (3.6) and (3.7) give necessary and sufficient conditions for $(\theta, l)$ to satisfy $(3.4)$ namely

$$
B_{n}\left(\begin{array}{l}
\theta_{n} \\
l_{n}
\end{array}\right)=\left(\begin{array}{l}
0 \\
0
\end{array}\right) \quad \text { at } r=R,
$$

where

$$
B_{n}=\left[\begin{array}{cc}
(m+2) d / d r-2 / r & \rho_{n} t^{1 / 2} m r \\
-\rho_{n} t^{1 / 2} & r d / d r
\end{array}\right]
$$

and $\left(\theta_{n}, l_{n}\right)$ is a linear combination of $\left(\theta_{n i}, l_{n i}\right), i=1,2$.

Since we desire a non-trivial solution, $\left(\theta_{n}, l_{n}\right)$ must be non-zero for at least one $n$. We therefore conclude, with the aid of Proposition 3.1, that a necessary and sufficient condition for the existence of a solution to (3.2)-(3.5) is that

$$
\operatorname{det}\left\{\left.B_{n}\left(\begin{array}{c}
\theta_{n 1} \\
l_{n 1}
\end{array}\right)\right|_{r=R} ;\left.\quad B_{n}\left(\begin{array}{c}
\theta_{n 2} \\
l_{n 2}
\end{array}\right)\right|_{r=R}\right\}=0
$$

for some $n$. 
If we substitute (3.7) into the last we conclude, with the aid of (3.8) and (1.2), that

$$
0=-4 t^{1 / 2} v\left(\rho_{n} R\right)+2\left(t^{1 / 2}-t^{-1 / 2}\right)-\left(t^{1 / 2}+t^{-1 / 2}\right)\left(m+2-\frac{t m}{e_{2}^{2}}\right) v\left(\rho_{n} R e_{2}\right),
$$

where $v(r) \equiv r I_{0}(r) / I_{1}(r)$.

We now prove that for any given value of $\rho=\rho_{n} R=n \pi R / L$ equation (3.10) is satisfied by a unique $\lambda \in(0,1)$.

THEOREM 3.2. For each $\rho>0$ there exists a unique $\lambda(\rho)$ such that

$$
H(\rho, \lambda(\rho))=0,
$$

where

$$
\begin{aligned}
H(\rho, \lambda) & \equiv v(\rho f)-R(\rho, \lambda) \\
R(\rho, \lambda) & \equiv \frac{4 t}{(1+t)^{2}} f^{2} v(\rho)-\frac{2(t-1)}{(1+t)^{2}} f^{2}, \\
f^{2} & \equiv e_{2}^{2}=1+(t-1) \frac{m+1}{m+2}, \quad t \equiv \lambda^{-(3 m+2) /(m+1)} .
\end{aligned}
$$

Moreover $\lambda:(0, \infty) \rightarrow(0,1)$ and is continuously differentiable in $\rho$.

Proof. Existence ${ }^{9}$. Fix $\rho>0$ and consider $H$ as a function of $\lambda$. We prove $H=0$ has a root $\lambda$ in the interval $(0,1)$ by showing

$$
\frac{\partial H}{\partial \lambda}(\rho, 1)>0, \quad \operatorname{Lim}_{\lambda \rightarrow 0^{+}} H(\rho, \lambda)=+\infty
$$

(existence clearly follows from (3.13), $H(\rho, 1)=0$, and the continuity of $H$ ).

To prove (3.13), we first note that $H$ is a function of $\lambda$ thru the variable $t$ and hence

$$
\frac{\partial H}{\partial \lambda}(\rho, 1)=\left.\left(\frac{\partial H}{\partial t} \frac{d t}{d \lambda}\right)\right|_{\lambda=1}=\left.\frac{-(3 m+2)}{m+1}\left(\frac{\partial H}{\partial t}\right)\right|_{t=1} .
$$

It thus suffices to show that

$$
\left.\frac{\partial H}{\partial t}\right|_{t=1}<0 .
$$

A straightforward computation, involving (3.12), yields (at $t=1$ )

$$
\frac{\partial H}{\partial t}=\frac{1}{2} \frac{m+1}{m+2} \rho \dot{v}(\rho)-\frac{m+1}{m+2} v(\rho)+\frac{1}{2},
$$

and hence by $(1.5)_{1}$ we find that (at $\left.t=1\right)$

$$
\frac{\partial H}{\partial t}=\frac{1}{2} \frac{m+1}{m+2}\left[\rho^{2}+\frac{m+2}{m+1}-v^{2}(\rho)\right] .
$$

Equation (3.14) now follows from (3.15) and Proposition 1.1(iii).

\footnotetext{
${ }^{9}$ The existence portion of this proof is a special case of the existence result presented in [9]. We have decided to re-present it since the special material used in this paper allows us to greatly simplify the proof.
} 
To prove (3.13) $)_{2}$ we note that as $\lambda \rightarrow 0^{+}, t \rightarrow \infty$, and hence $f^{2} / t \rightarrow(m+1) /(m+2)$. It then follows from (3.12) $)_{2}$ that $R$ is bounded as $\lambda \rightarrow 0^{+}$. But by Proposition 1.1, $\dot{v}$ and $\ddot{v}$ are strictly positive. Thus $v(\rho f) \rightarrow+\infty$ and hence by (3.12) 1 we conclude (3.13) $)_{2}$. This completes the existence portion of the proof.

Uniqueness. By (3.12) ${ }_{3}$ we see that $f$ is strictly monotone in $\lambda$ so that we could just as well consider (3.10) as a relation between $\rho$ and $f$ and seek roots $f$ of $H$. Thus we rewrite (3.11) and (3.12) as

$$
\tilde{H}(\rho, f)=0
$$

where

$$
\begin{aligned}
& \tilde{H}(\rho, f)=v(\rho f)-\tilde{R}(\rho, f), \\
& \tilde{R}(\rho, f)=\frac{2(m+1) f^{2}}{\left[(m+2) f^{2}+m\right]^{2}}\left\{(m+2)(2 v-1) f^{2}+(m+2-2 v)\right\},
\end{aligned}
$$

and $v \equiv v(\rho)$.

We will show that $\tilde{H}$ is a strictly convex function of $f$ for $f \geqslant 1$. This allows us to conclude that there is at most one $f$ satisfying $(3.16)$ (since $\tilde{H}(\rho, 1)=0$ ) and hence at most one $\lambda$ satisfying (3.11).

Since $\ddot{v}$ is strictly positive (cf. Proposition 1.1), it suffices to show that

$$
\frac{\partial^{2} \tilde{R}}{\partial f^{2}}<0 \text { for all } f \geqslant 1
$$

By direct calculation we find that

$$
\frac{\partial^{2} \tilde{R}}{\partial f^{2}}=\frac{4(m+1)}{\left[(m+2) f^{2}+m\right]^{4}}\left\{a(m) f^{4}+b(m) f^{2}+c(m)\right\},
$$

where

$$
\begin{aligned}
& a(m)=-3(m+2)^{2}[(4 m+2) v-3 m-2], \\
& b(m)=m(m+2)[(12 m+16) v-14 m-16], \\
& c(m)=m^{2}(m+2-2 v) .
\end{aligned}
$$

We show that the quantity in braces is strictly negative for all $f \geqslant 1$. Put $x=f^{2}$ and define $Q(x)$ to be the quadratic in braces. It clearly suffices to show that both $Q(1)$ and $\dot{Q}(1)$ are strictly negative.

Clearly

$$
Q(1)=-v\left(16 m^{2}+40 m+24\right)-\left(4 m^{3}-28 m-24\right)
$$

and

$$
\dot{Q}(1)=-(m+2)\left[\left(12 m^{2}+44 m+24\right) v-\left(4 m^{2}+32 m+24\right)\right] .
$$

The desired result now follows from $v \geqslant 2$ (cf. Proposition 1.1 and equation $(1.5)_{2}$ ). This establishes the uniqueness of the root $\lambda$ of (3.11). 
Differentiability. By the implicit function theorem the continuous differentiability of $f(\rho)$, and hence $\lambda(\rho)$, follows provided we know that

$$
\frac{\partial \tilde{H}}{\partial f}(\rho, f(\rho)) \neq 0 .
$$

Since $\tilde{H}$ is zero at $f=1$ and $f=f(\rho)$, we can apply Rolle's Theorem to conclude that there exists an $f^{*} \in(1, f(\rho))$ such that $\tilde{H}_{f}\left(\rho, f^{*}\right)=0$. But $\tilde{H}$ is strictly convex and hence $\tilde{H}_{f}$ is a strictly increasing function of $f$, thus

$$
\frac{\partial \tilde{H}}{\partial f}(\rho, f(\rho))>0 .
$$

This completes the proof.

4. Discussion. Further results. In this section we derive further information concerning the dependence of $\lambda$ on $\rho(=n \pi R / L)$. We show that the function $\lambda=\lambda(\rho)$ is strictly decreasing for large $\rho$ and strictly increasing for small $\rho$, and that it has no local minima. These results along with those of the previous section lead us to the following conclusions.

For a given geometry (ratio $R / L$ ) and a given mode number $n$ there is a (unique) corresponding $\lambda_{n} \in(0,1)$ such that the linear problem (3.2)-(3.5) has a nontrivial solution. Letting

$$
\lambda_{\mathrm{cr}}=\sup \left\{\lambda_{n}: n=1,2,3 \ldots\right\}
$$

and noting that $\lambda_{\mathrm{cr}}=\lambda_{n}$ for some finite value of $n$, we find that for $\lambda \in\left(\lambda_{\mathrm{cr}}, 1\right]$ the nonlinear homogeneous solution $\mathbf{f}_{\lambda}$ (cf. (2.4)) is stable with respect to axisymmetric (barrelling) perturbations and that at $\lambda=\lambda_{\mathrm{cr}}$ this stability breaks down.

In addition, the absence of local minima in the function $\lambda(\rho)$ implies that at most two modes can occur simultaneously as nontrivial solutions of the linear problem. Clearly, it would be of interest to know precisely which value of $n$ yields the largest $\lambda_{n}$. To date we have been unable to determine this analytically. However, the results of this section do tell us that for sufficiently thick cylinders $(R / L \gg 1), n=1$ yields the largest $\lambda$ while for sufficiently thin cylinders $n=1$ does not yield the largest $\lambda$.

We first investigate the asymptotic behavior of $\lambda(\rho)$ near zero and infinity.

Proposition 4.1.

(i)

$$
\underset{\rho \rightarrow \infty}{\operatorname{Liminf}} f(\rho)>1 ;
$$

(ii)

(iii)

$$
f(\rho) \text { is bounded as } \rho \rightarrow \infty \text {; }
$$

$$
\operatorname{Lim}_{\rho \rightarrow 0} f(\rho)=+\infty
$$

$$
\underset{\rho \rightarrow 0}{\operatorname{Liminf}} \rho f(\rho)>0 .
$$


Proof. Consider the function $h(f) \equiv v(\rho f)$. If we apply the mean value theorem to $h$ we find that

$$
v(\rho f)=v(\rho)+\rho \dot{v}\left(\rho f^{*}\right)(f-1)
$$

where

$$
1 \leqslant f^{*} \leqslant f
$$

If we then substitute (4.1) into (3.12) and divide the result by $\rho(t-1)$ we conclude, with the aid of $(3.12)_{3}$, that

$$
\frac{v(\rho)}{\rho} \frac{(m+2)(t-1)-4 t(m+1)}{(m+2)(1+t)^{2}}+\frac{\dot{v}\left(\rho f^{*}\right)}{(f+1)} \frac{(m+1)}{(m+2)}=-\frac{2 f^{2}}{\rho(1+t)^{2}} .
$$

To prove (i) we assume, for the sake of contradiction, that there is a sequence $\rho_{i} \rightarrow \infty$ such that $f\left(\rho_{i}\right) \rightarrow 1$. If we let $\rho=\rho_{i} \rightarrow \infty$ in (4.3) we find, with the aid of (3.12) $)_{3},(4.2)$, and (1.6), that (4.3) reduces to

$$
\frac{-4(m+1)}{(m+2) 2^{2}}+\frac{1}{2} \frac{(m+1)}{(m+2)}=0 .
$$

This is clearly impossible for any $m>0$. Thus we arrive at (i).

To prove (ii) we assume, again for the sake of contradiction, that there is a sequence $\rho_{i} \rightarrow \infty$ such that $f_{i} \equiv f\left(\rho_{i}\right) \rightarrow \infty$. If we divide (3.17) by $\rho f$ we find that

$$
\frac{v(\rho f)}{\rho f}=\frac{2(m+1)}{\left[(m+2) f^{2}+m\right]^{2}}\left\{(m+2)\left(2 \frac{v}{\rho}-\frac{1}{\rho}\right) f^{3}+\left(\frac{m+2}{\rho}-\frac{2 v}{\rho}\right) f\right\},
$$

where $v \equiv v(\rho)$. Letting $\rho=\rho_{i} \rightarrow \infty$ we find, by (1.6), that the left-hand side of the last equation approaches one while the right-hand side goes to zero. Thus no such sequence can exist. This proves (ii).

To prove (iii) we multiply (4.3) by $\rho$ and let $\rho \rightarrow 0$ to conclude, with the aid of $(3.12)_{3}$ and $(1.6)_{2}$, that $(4.3)$ reduces to

$$
\operatorname{Lim}_{\rho \rightarrow 0} \frac{t(2 m+1)+(m+1)}{(m+2)(1+t)^{2}}=0,
$$

where $t \equiv t(\rho)$. Now Theorem 3.2 implies that $t(\rho)$ is a $C^{1}$ function for $\rho \in(0, \infty)$ and moreover that $t(\rho) \in(1, \infty)$ for the same $\rho$. Clearly, the only way (4.4) can be satisfied is for $t(\rho)$, and hence $f(\rho)$, to become infinite. This proves (iii).

To prove (iv) we assume, once more for the sake of contradiction, that there is a sequence $\rho_{i} \rightarrow 0$ such that $\rho_{i} f\left(\rho_{i}\right) \rightarrow 0$. If we let $\rho=\rho_{i} \rightarrow 0$ in (3.12) we find, with the aid of $(1.5)_{2}$, and (iii) of this proposition, that

$$
2-8 \frac{m+1}{m+2}+2 \frac{m+1}{m+2}=0 .
$$

This is clearly impossible for any $m>0$. Thus no such sequence can exist. This concludes the proof of the proposition. 
To analyze the behavior of $d \lambda / d \rho$ near zero and infinity, we differentiate (3.11) with respect to $\rho$ to conclude

$$
\frac{d \lambda}{d \rho}=-\frac{\partial H}{\partial \rho}(\rho, \lambda(\rho)) / \frac{\partial H}{\partial \lambda}(\rho, \lambda(\rho)) .
$$

In view of (3.12) ${ }_{3}$ and (3.19) we find that $H_{\lambda}(\rho, \lambda(\rho))$ is strictly negative and hence that

$$
\operatorname{sgn} \frac{d \lambda}{d \rho}=\operatorname{sgn} \frac{\partial H}{\partial \rho}(\rho, \lambda(\rho)) \text {. }
$$

THEOREM 4.2. There exists a $\rho_{0}>0$ such that

$$
\frac{d \lambda}{d \rho}>0 \text { for } \rho \in\left(0, \rho_{0}\right) \text {. }
$$

Proof. In view of (4.5) we prove that $H_{\rho}(\rho, \lambda(\rho))$ is strictly positive for $\rho \in\left(0, \rho_{0}\right)$. If we differentiate (3.12) with respect to $\rho$ we find that

$$
\frac{1}{f(\rho)} \frac{\partial H}{\partial \rho}(\rho, \lambda(\rho))=\dot{v}(\rho f(\rho))-\frac{4 t(\rho) f(\rho)}{(1+t(\rho))^{2}} \dot{v}(\rho) .
$$

We note that by Proposition 1.1, $\dot{v}$ is strictly positive on $(0, \infty)$. Thus if we let $\rho \rightarrow 0$ in (4.7) we discover, with the aid of (3.12) 3 , and Proposition 4.1(iv), that the right-hand side of (4.7) is strictly positive for sufficiently small $\rho$. This gives the desired result.

We next get more precise information on the asymptotic behavior of $\lambda(\rho)$ near infinity. Proposition 4.3. As $\rho \rightarrow \infty \lambda(\rho)$ tends to the value $\tau^{-(m+1) /(3 m+2)}$ where $\tau$ is the unique root (greater than one) of the cubic polynomial

$$
\tau^{3}-\frac{11 m+6}{m+2} \tau^{2}-5 \tau-1 .
$$

Proof. For brevity we put

$$
T_{1}=\frac{4 t}{(1+t)^{2}}, \quad T_{2}=\frac{2(t-1)}{(1+t)^{2}}
$$

and substitute the asymptotic estimate (1.6), into (3.12) to get

$$
f\left[1-f T_{1}\right]=-\frac{1}{2 \rho}\left[1-T_{1} f^{2}+2 T_{2} f^{2}\right]-\frac{1}{\rho}\left[a(\rho f)-f^{2} T_{1} a(\rho)\right] .
$$

If we let $\rho \rightarrow \infty$ in (4.9) we find, with the aid of $(3.12)_{3},(1.6)_{1}$, and Proposition 4.1(ii), that

$$
\operatorname{Lim}_{\rho \rightarrow \infty} f(\rho) T_{1}(\rho)=1 \text {. }
$$

From the last and the definitions of $f$ and $T_{1}$, we discover that $t(\rho)$ tends to a nonnegative root of the quartic polynomial

$$
(\tau-1)\left(\tau^{3}-\frac{11 m+6}{m+2} \tau^{2}-5 \tau-1\right) .
$$

We note that a simple computation shows that both the cubic polynomial and its first derivative are negative at one and hence that the polynomial has a unique root greater than one. Proposition 4.1(i) and (3.12) $)_{3}$ then allow us to conclude that $t(\rho)$ tends toward this root. This concludes the proof. 
We next analyze the behavior of $d \lambda / d \rho$ near infinity.

THEOREM 4.4. There exists a $\rho_{1}>0$ such that $\dot{\lambda}(\rho)<0$ for $\rho \in\left(\rho_{1}, \infty\right)$.

Proof. In view of (4.5) we prove that $H_{\rho}(\rho, \lambda(\rho))$ is strictly negative for $\rho \in\left(\rho_{1}, \infty\right)$. If we differentiate (3.12) with respect to $\rho$ we find, with the aid of (4.8), that

$$
\rho \frac{\partial H}{\partial \rho}=\rho f \dot{v}(\rho f)-T_{1} f^{2} \rho \dot{v}(\rho) .
$$

Substituting the asymptotic expansion $(1.6)_{2}$ into the last expression yields

$$
\rho \frac{\partial H}{\partial \rho}=\rho f\left[1-f T_{1}\right]+\left[\rho f b(\rho f)-f^{2} T_{1} \rho b(\rho)\right] .
$$

If we combine the last expression and (4.9) we find that

$$
\rho \frac{\partial H}{\partial \rho}(\rho, \lambda(\rho))=-\frac{1}{2}\left[1-T_{1}(\rho) f(\rho)^{2}+2 T_{2}(\rho) f(\rho)^{2}\right]+c(\rho),
$$

where

$$
c(\rho)=\left(\rho f b(\rho f)-\rho f^{2} T_{1} b(\rho)\right)-\left(a(\rho f)-f^{2} T_{1} a(\rho)\right) .
$$

It is clear from Proposition 4.3 and (1.6) that $c(\rho)$ tends to zero as $\rho$ becomes infinite. To evaluate the expression in square brackets on the right-hand side of (4.11) we use (4.10), (4.8), and Proposition 4.3 to conclude that

$$
\operatorname{Lim}_{\rho \rightarrow \infty} \rho \frac{\partial H}{\partial \rho}(\rho, \lambda(\rho))=\frac{-1}{8 \tau^{2}}(3 \tau+1)(\tau-1),
$$

where $\tau$ is the unique root (greater than one) of the cubic polynomial constructed in Proposition 4.3. This yields the desired result.

Finally, we show that the function $\lambda(\rho)$ has no local minima.

THEOREM 4.5. If $\dot{\lambda}(\rho)=0$ for some $\rho>0$ then $\ddot{\lambda}(\rho)<0$ at the same $\rho$.

Proof. We first note that by (3.19) and the implicit function theorem, the function $\lambda(\rho)$ is $C^{2}$. If we differentiate $H(\rho, \lambda(\rho))=0$ twice with respect to $\rho$ and let $\dot{\lambda}=0$ we find that

$$
H_{\rho}=0, \quad \ddot{\lambda}=-H_{\rho \rho} / H_{\lambda} .
$$

By (3.12) and (3.19) it therefore suffices to prove that $H_{\rho \rho}<0$ whenever $H_{\rho}=0$.

If we differentiate (3.12) with respect to $\rho$ we find that

$$
H_{\rho}=f \dot{v}(\rho f)-\frac{4 t}{(1+t)^{2}} f^{2} \dot{v}(\rho), \quad H_{\rho \rho}=f^{2} \ddot{v}(\rho f)-\frac{4 t}{(1+t)^{2}} f^{2} \ddot{v}(\rho) .
$$

Thus, using $H_{\rho}=0$, we conclude that

$$
H_{\rho \rho}=\rho^{-1} \dot{v}(\rho f)[w(\rho f)-w(\rho)],
$$

where

$$
w(t) \equiv t \ddot{v}(t) / \dot{v}(t)
$$

Now, by Proposition $1.1, \dot{v}$ is strictly positive, while by the proposition in the Appendix $w$ is strictly decreasing. Thus, since $f>1$, we conclude that $H_{\rho \rho}<0$. This concludes the proof. 
5. Numerical results. In Fig. 1 we plot the curve $\lambda(\rho)$ that solves $H(\rho, \lambda(\rho))=0$ when $m=13.3$. Other values of $m$ between .1 and 1000 gave essentially the same picture.

If one wants to find the mode and loading value at which instability first occurs, first fix values of $R$ and $L$ and plot the points $\rho_{n}=n \pi R / L$ (for $n=1,2, \ldots$ ) on the $\rho$ axis. Then plot the points $\left(\rho_{n}, \lambda\left(\rho_{n}\right)\right)$ using the graph. The highest point $\lambda\left(\rho_{n}\right)$ determines both the eigenmode $n$ and the first $\lambda$. We note that if $\pi R / L>.59$ then $n=1$ is the first eigenmode to bifurcate. If $\pi R / L \in(.32, .59)$ then $n=2$ comes first and if $\pi R / L \in(.23, .32)$ then $n=3$ comes first, etc.

Remark. It follows from Theorem 4.2 and Theorem 4.4 (or the above graph) that there exists geometries (ratios $R / L$ ) such that the largest $\lambda$, at which instabilities occur, can have two eigenmodes associated with it. Since the maximum value of $\lambda, \lambda_{\max }$, and the asymptotic value of $\lambda, \lambda_{\text {asy }}$, are so close to .45 for any $R / L$ the corresponding value of $\lambda_{\text {cr }}$ will always be near .45 , i.e. instability always occurs for $\lambda$ near .45 .

Remark. We note that our numerical results are not consistent with the experiments of Beatty \& Hook [2] and Beatty \& Dadras [1]. While our largest value of $\lambda_{\mathrm{cr}}$ never exceeded .45 , their smallest value was never less than .70 . The most likely reason for this is that our choice of constitutive relation $((2.1))$ is not appropriate for solid rubbers.

Appendix. We prove a result concerning the function $w(t) \equiv t \ddot{v}(t) / \dot{v}(t)$.

Proposition. $\dot{w}(t)$ is strictly negative for $t \in(0, \infty)$.

Proof. If we differentiate (1.5), we find that

$$
\frac{1}{2} w(t)=\frac{t}{\dot{v}(t)}-v(t)+\frac{1}{2}
$$

and hence that

$$
\frac{1}{2} \dot{w}(t)=-2[u(t)-v(t)] / \dot{v}(t),
$$

where

$$
u(t) \equiv\left[2 t+\dot{v}^{3}(t)\right] / 2 \dot{v}(t) .
$$

To finish the proof we will show that

$$
u(t)>v(t) \text { for all } t>0 .
$$

To show that (A.1) is true near $t=0$ define

$$
q(t) \equiv \dot{v}(t)[u(t)-v(t)] .
$$

Then by differentiating (1.5), and (A.2) a sufficient number of times one can show that

$$
\dot{v}(0)=0, \quad \ddot{v}(0)=\frac{1}{2}, \quad \ddot{v}(0)=0, \quad v^{(4)}(0)=\frac{-1}{4}
$$

and hence that

$$
q(0)=0, \quad \dot{q}(0)=0, \quad \ddot{q}(0)=0, \quad \ddot{q}(0)=\frac{1}{8} .
$$

We conclude that (A.1) holds in a neighborhood of zero.

Next, assume for the sake of contradiction that (A.1) is not true on $(0, \infty)$ and define

$$
s \equiv \inf \{t \in(0, \infty): u(t)=v(t)\} .
$$




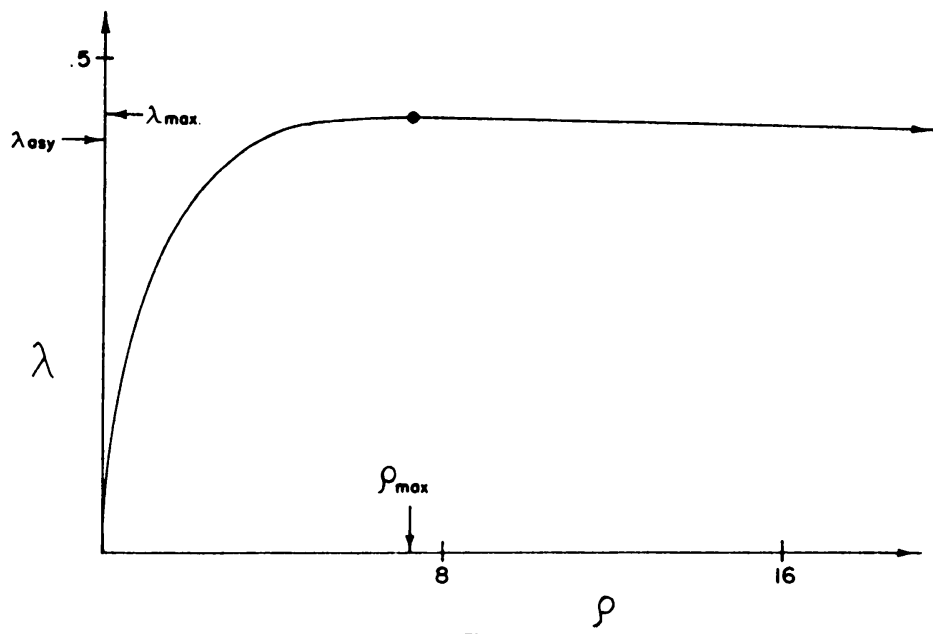

Fig. 1.

It is clear that $\dot{v}(s) \geqslant \dot{u}(s)$. However

$$
\begin{aligned}
\dot{u}(s)-\dot{v}(s) & =\left[\dot{v}^{3} \ddot{v}+(\dot{v}-s \ddot{v})-\dot{v}^{3}\right] / \dot{v}^{2} \\
& =\left[\dot{v}^{3} \ddot{v}+(2 v \dot{v}-2 s)-\dot{v}^{3}\right] / \dot{v}^{2} \\
& =\dot{v}(s) \ddot{v}(s)>0,
\end{aligned}
$$

a contradiction. Thus no such $s$ can exist.

Acknowledgement. The authors would like to thank Professor J. L. Ericksen for suggesting we pursue this problem and Professor E. Sternberg for his helpful comments. The authors would also like to thank J. W. Blakeman and A. W. Mifflin for producing extensive numerical data used in Sec. 5. This research was supported in part by the Institute for Mathematics and its Applications, University of Minnesota and by the National Science Foundation under grant MCS-8102831.

\section{REFERENCES}

[1] M. F. Beatty and P. Dadrus, Some experiments on the elastic stability of highly elastic bodies, Int. J. Eng. Sci. 14, 233-238 (1976)

[2] M. F. Beatty and D. E. Hook, Some experiments in the stability of circular rubber bars under end thrust, Int. J. Solids Structures 4, 623-635 (1968)

[3] P. J. Blatz and W. L. Ko, Application of finite elasticity theory to the deformation of rubbery materials, Trans. Soc. Rheology 6, 223-251 (1962)

[4] P. Bridgman, The compression of sixty-one solid substances to $25,000 \mathrm{~kg} / \mathrm{cm}^{2}$, determined by a new rapid method, Proc. Am. Acad. Arts Sci. 76, 9-24 (1945)

[5] I. W. Burgess and M. Levinson, The instability of slightly compressible rectangular solids under biaxial loadings, Int. J. Solids Struc. 8, 133-148 (1972)

[6] E. T. Copson, Asymptotic expansions, Cambridge Univ. Press, Cambrige (1965)

[7] K. N. Sawyers and R. S. Rivlin, Stability of a thick elastic plate under thrust, J. Elasticity 12, 101-125 (1982)

[8] K. N. Sawyers, Stability of a thick neo-Hookean plate, Proc. IUTAM Sym. Finite Elasticity (D. E. Carlson and R. T. Shield, eds.), Martinus Nijhoff, Boston (1982)

[9] H. C. Simpson and S. J. Spector, On barrelling instabilities in finite elasticity, J. Elasticity. In press

[10] __ Some monotonicity results for ratios of modified Bessel functions, Quart. Appl. Math. This issue

[11] E. W. Wilkes, On the stability of a circular tube under end thrust, Quart. J. Mech. Appl. Math., 8, 88-100 (1955) 